$7-1-2021$

\title{
Adenoid cystic carcinoma of external auditory canal: A rare disease
}

Abdul Basit Shah Vardag

Muhammad Hassan Danish

Mohammad Sohail Awan

Muhammad Usman Tariq

Omaima Anis Bhatti

See next page for additional authors

Follow this and additional works at: https://ecommons.aku.edu/ pakistan_fhs_mc_surg_otolaryngol_head_neck

Part of the Otolaryngology Commons, Otorhinolaryngologic Diseases Commons, and the Pathology Commons 


\section{Authors}

Abdul Basit Shah Vardag, Muhammad Hassan Danish, Mohammad Sohail Awan, Muhammad Usman

Tariq, Omaima Anis Bhatti, and Muhammad Hammad Deewani 


\section{CASE REPORT}

\section{Adenoid cystic carcinoma of external auditory canal: A rare disease}

Abdul Basit Vardag, ${ }^{1}$ Muhammad Hassan Danish, ${ }^{2}$ Muhammad Sohail Awan, ${ }^{3}$ Muhammad Usman Tariq, ${ }^{4}$ Omaima Anis Bhatti, ${ }^{5}$ Muhammad Hammad6

\begin{abstract} extension. The malignancy needs to be addressed with an aggressive approach surgically with adequate marginal clearance. The role of radiation is debatable considering the outcomes in the limited data. Here we present the case of a 35-year-old female who came in with otalgia and otorrhea associated with a mass in the external auditory canal. After biopsy and imaging, a diagnosis of adenoid cystic carcinoma was made and the patient was managed accordingly.
\end{abstract}

External auditory canal, like other epithelialized surfaces is predisposed to malignancies such as Squamous cell carcinomas (SCC), melanomas and adenocarcinomas. In this background, malignancies like adenoid cystic carcinoma (ACC) are a rare occurrence and need to be thoroughly evaluated both locally and for distant

Keywords: Adenoid cystic carcinoma, External auditory canal, Neck dissection.

DOI:https://doi.org/10.47391/JPMA.572

\section{Introduction}

External ear malignancies are an oddity with squamous cell carcinoma (SCC) being the most commonly seen malignancy. ${ }^{1}$ In this background, the incidence of adenoid cystic carcinoma (ACC) is worth mentioning. ACC has an inclination towards the oral cavity and the bordering structures, i.e. oropharynx, salivary glands and nasopharynx with less than $0.2 \%$ of cases seen in the external auditory canal (EAC).2,3 Because of limited amount of literature available, evidence-based management is not well defined. ${ }^{1}$ Here, we have endeavoured to put forth our experience and highlight the major steps of management of this rare entity.

1-3,6Department of Otolaryngology, Head and Neck Surgery, ${ }^{4}$ Department of Pathology, Aga Khan University Hospital, Karachi, 53rd Year MBBS Student, Medical College, Aga Khan University, Karachi, Pakistan.

Correspondence: Muhammad Hassan Danish. Email: hassan.danish@aku.edu

\section{Case Report}

A 35-year-old lady with no co-morbids presented to the Ear Nose and Throat (ENT) clinic of Aga Khan University Hospital, Karachi on 26th July, 2019. She complained of right otalgia and episodic discharge for the past six weeks. The otorrhea was serous and clear. There was no symptom of hearing loss or vertigo. The past history was

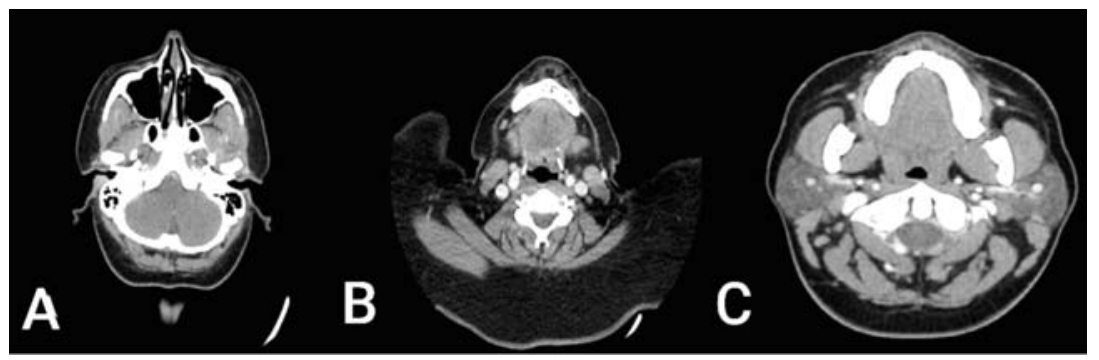

Figure-1: A) A soft tissue density visible present along the posterior aspect of the external auditory canal without any erosion into the temporal bone. B) Nodal involvement. () No signs of soft tissue anterior invasion into the parotid gland or nodal involvement.

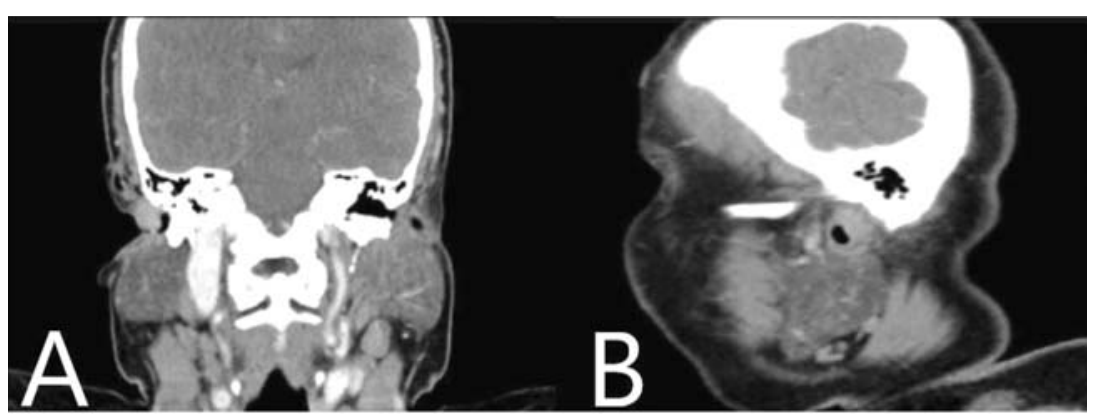

Figure-2: A) Coronal view showing enhancing lesion in right external auditory canal arising from superior canal. B) Sagittal view showing soft tissue lesion partially obstructing the lumen of external auditory canal.

also unremarkable. On examination, her right ear was tender to touch and otoscopic examination revealed a firm and non-fluctuant swelling arising from the posterosuperior aspect of the EAC. It was placed in the outer one-third of the EAC with scant crusting of discharge. Microscopic examination of the ear was done and tissue was obtained by punch biopsy. This histopathology was reported as adenoid cystic carcinoma.

A contrast enhanced Computed Tomography (CT) study of the temporal bone and neck was done. It showed a 


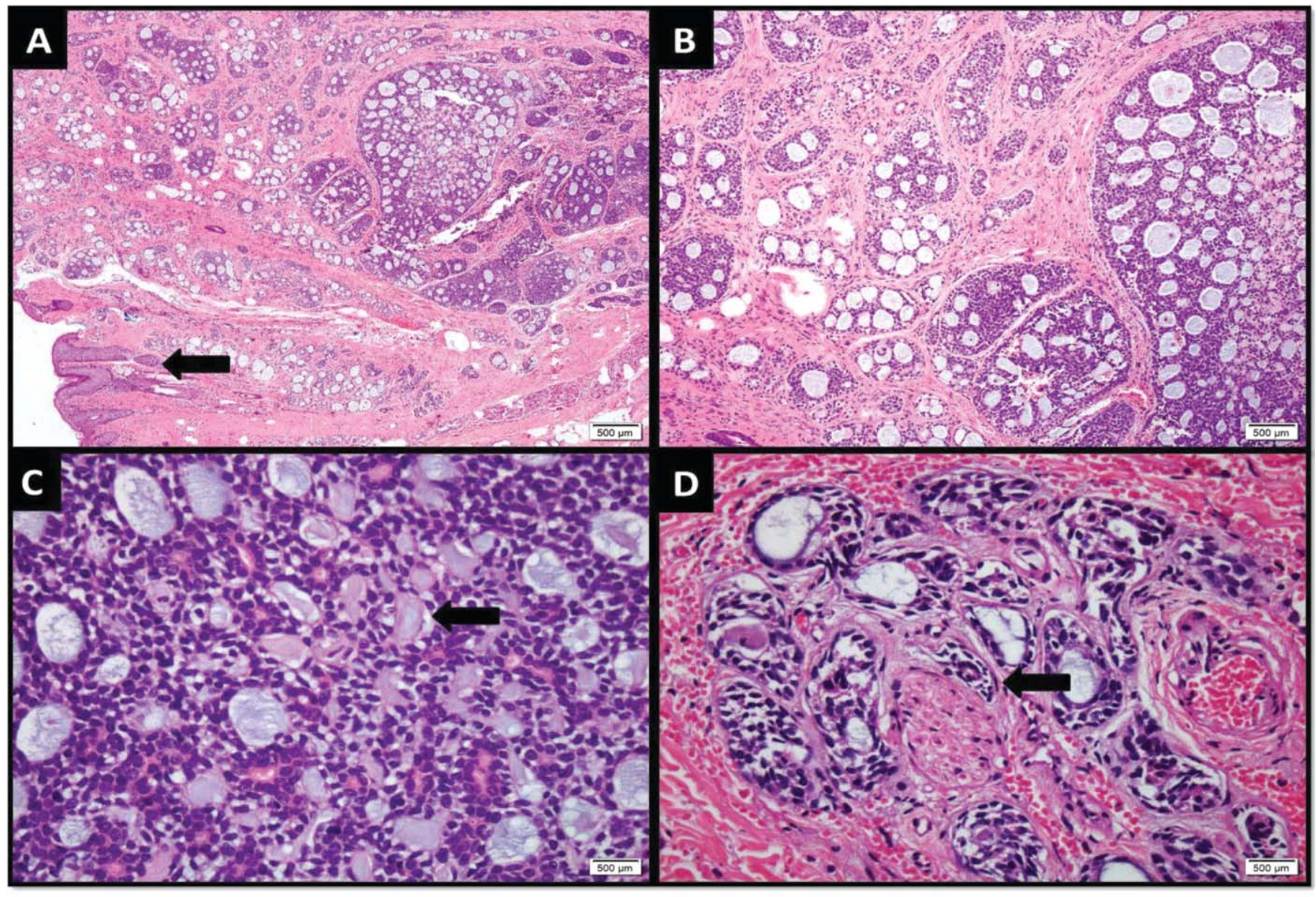

Figure-3: A) Skin covered tissue exhibiting a neoplastic lesion involving the dermis and the subcutaneous adipose tissue. Arrow is pointing towards epidermis. B) Nodules and nests with frequent small cystic spaces and tubule formation. () Tumour cells are basaloid and uniform. Eosinophilic basement lamina-like material is seen in the lumina (arrow). D) Perineural invasion (arrow).

partly defined enhancing soft tissue density lesion in the lateral aspect of the EAC on the right side which measured $11 \times 12 \times 13 \mathrm{~mm}$ in antero-posterior, transverse and craniocaudal dimensions respectively (Figure-1 and 2). There was no underlying infiltration into the mastoid part of the right temporal bone or extension anteriorly into the parotid gland.

As the tumour was limited to the EAC and there was lack of bony erosion, the tumour was staged as T1 using University of Pittsburgh TNM staging for EAC carcinoma ${ }^{4}$. Right level II showed a $10 \mathrm{~mm}$ lymph node. The patient was planned for surgery.

The patient underwent wide local excision of tumour with right supraomohyoid neck dissection (Level I, II and III) on 31st July, 2019. Intra-operatively a right sided $1.5 \times 1.5 \mathrm{~cm}$ mass involving the posterior wall of the EAC and extending to superior canal was encountered. It was starting from the cartilaginous canal and extending to the bony canal medially. The lesion was excised. The underlying periosteum over the bony canal was healthy. Frozen sections were sent intra-operatively from medial, lateral, superior and inferior edges after excision, which were all reported to be negative.

Histologically, the primary specimen consisted of a greywhite firm tumour measuring $1.5 \times 1 \times 1 \mathrm{~cm}$ lesion grossly. On microscopic examination, the dermis and the subcutaneous adipose tissue were infiltrated by a neoplastic lesion composed of nodules and nests with frequent small cystic spaces and tubule formation (Figure-3A and B). These basaloid neoplastic cells had scant cytoplasm and round to oval uniform nuclei with fine chromatin, inconspicuous nucleoli and scattered mitotic figures. Lumina of the cystic spaces and tubules contained eosinophilic basement lamina like material (Figure-3C). Perineural invasion was also identified (Figure-3D). Skin ulceration and lymphovascular invasion were not identified. All 25 lymph nodes were negative for 
tumour metastasis. Pathologically the tumour was staged as pT1, N0 with superior and inferior margins being the closest margins. Patient was discharged and advised for monthly follow-ups. The patient was last seen in March 2020 at the clinic, she is disease free and doing well.

\section{Discussion}

The presentation of ACC in the EAC is usually of severe otalgia, so much so that sometimes needle biopsy can be difficult to perform. ${ }^{5}$ In our case the primary complaint was otalgia but not as severe as reported previously. We were able to take a punch biopsy under local anaesthesia in the clinic. Only $10-15 \%$ of the tumours of the ear are housed in the EAC with a major predominance of SCC. ${ }^{3}$ Other than SCC, differential diagnosis includes basal cell carcinoma, adenocarcinoma, ACC, melanomas and sarcomatous lesions of the external canal. ${ }^{1}$ Adenoid cystic carcinoma in the EAC is primarily a malignancy of the secretory tissue, the ceruminous glands in the external auditory canal. ${ }^{5}$ The origin of the tissue has been a source of controversy.

The salivary gland analogue was explained by Billroth, where the lesion was referred to as cylindroma. It was categorised as a benign lesion in the salivary glands initially. The overall prevalence ranged from $4-15 \% .^{2}$ Histologically, ACC was shown to have three variants of different prognostic implications in the salivary gland tumours i.e. tubular, cribriform and solid. Though, similarities have been observed between the two analogues including local tissue extension and perineural, one study found it to be $80 \%$. A detailed account of the correlation between the variants with their prognosis has not been established for EAC. ${ }^{5}$ The location of ACC, on rare occasions was found in the EAC which has been reported to have a wide spectrum of invasion.

The presentation of ACC is most commonly in the form of otalgia, otorrhea, hearing loss and a mass present in the external canal. This progresses to symptoms associated with invasion, both local and distant. These include extension into the surrounding tissue mainly intracranial, into the parotid gland and neck nodes to distant tissue like pulmonary metastasis. ${ }^{5-7}$ ACC is associated with early direct parotid extension even in stage 1 lesions. ${ }^{8}$

After the usual presentation, a cytological assessment is warranted. On smear, tri-dimensional aggregates, arranged uniformly into nests are observed with a scant amount of cytoplasm and nuclei showing hyperchromatic uptake. ${ }^{5}$ ACC does not have a separate classification for tumours of EAC and therefore, we have used University of Pittsburgh TNM staging. ${ }^{4}$

Management of ACC in the EAC is primarily based on surgeon's experience, as due to the limited number of cases and scarcity of literature, an evidence-based management guideline has not been established. ${ }^{1}$ Surgical management is predicated primarily on the extent of disease varying from local resection to lateral and sub-total temporal bone resection (LTBR and STBR, respectively). ${ }^{9}$ In our case, local resection with margin clearance was opted with unilateral supraomohyoid neck dissection. In this study, as there was no extension into the parotid on imaging, parotidectomy was not done. Post-operatively, radiation can be considered as an adjuvant therapy depending on the staging of the lesion, but the outcome is difficult to anticipate. Despite the available limited data, post-op radiation therapy has not yielded favourable results with a 5-year survival decline of 24\%.9 Chemotherapy has been considered for salivary gland tumours. The analogue and the response associated with ACC has been poor; consequently, but no substantial evidence regarding usage for External canal ACCs was seen. ${ }^{10}$ The disease is associated with an overall 5 -year survival of $70 \%$ with staging and margin clearance being the most significant prognostic factors. The survival ranges from $85 \%$ for $\mathrm{T} 1$ to $30 \%$ for T4 illness. Margin clearance has been reported to show an improvement of $35 \%$ in 5 -year survival rates. ${ }^{9}$

\section{Conclusion}

ACC of the EAC is primarily a rare malignancy of ceruminous tissue with local extension involving the parotids, neck nodes and intracranial structures. A greater index of suspicion must be employed with emphasis on imaging, both for local and distant spread. Eradication of the lesion should be taken up as aggressively as possible. More studies are needed to establish a management guideline.

Disclaimer: This manuscript has not been previously presented or published in any conference.

Conflict of Interest: This case report has no conflict of interest.

Funding Disclosure: No funding source was used in writing this manuscript.

\section{References}

1. Gidley PW. Managing malignancies of the external auditory canal. Expert RevAnticancer Ther. 2009; 9:1277-82.

2. Sigal R, Monnet O, De Baere T, Micheau C, Shapeero LG, Julieron $M$, et al. Adenoid cystic carcinoma of the head and neck: evaluation with MR imaging and clinical-pathologic correlation in 27 patients. Radiology. 1992; 184:95-101.

3. Paule MD, Fernandez-Gomez FJ, Llorent AA, Devis IA, Perez FP. Cylindroma of the external auditory canal: histopathology diagnostic. Otol Neurotol. 2010; 31:546-7.

4. Arriaga M, Curtin H, Hirsch BE, Takahashi H, Kamerer DB. Staging 
proposal for external auditory meatus carcinoma based on preoperative clinical examination and computed tomography findings. Ann Otol Rhinol Laryngol. 1990; 99:714-21.

5. Nayak SP, Walke VA, Helwatkar SB, Bobhate SK. Adenoid cystic carcinoma of the external auditory canal: report of two cases. Indian J Pathol Microbiol. 2009; 52:540-2.

6. Değirmenci B, Yücel A, Acar M, Albayrak R. Adenoid cystic carcinoma of the external auditory canal with pulmonary metastasis. Turk J Med Sci. 2004; 34:195-7.

7. De Lucia A, Gambardella T, Carra P, Motta G. A case of highly aggressive adenoid cystic carcinoma of the external auditory canal. Acta Otorhinolaryngol Ital. 2004; 24:354-56.

8. Choi JY, Choi EC, Lee HK, Yoo JB, Kim SG, Lee WS. Mode of parotid involvement in external auditory canal carcinoma. J Laryngol Otol. 2003; 117:951-4.

9. Gu FM, Chi FL, Dai CF, Chen B, Li HW. Surgical outcomes of 43 cases with adenoid cystic carcinoma of the external auditory canal. Am J Otol. 2013; 34:394-8.

10. Dodd RL, Slevin NJ. Salivary gland adenoid cystic carcinoma: a review of chemotherapy and molecular therapies. Oral Oncol. 2006; 42:759-69. 